\title{
Analyzing X-ray variability by linear state space models
}

\author{
M. König ${ }^{1}$ and J. Timmer ${ }^{2,3}$ \\ 1 Institut für Astronomie und Astrophysik - Astronomie, Universität Tübingen, Waldhäuser Str. 64, D-72076 Tübingen, \\ Germany \\ 2 Fakultät für Physik, Albert-Ludwigs-Universität, Hermann-Herder Str. 3, D-79104 Freiburg, Germany \\ 3 Freiburger Zentrum für Datenanalyse und Modellbildung, Albert Str. 26-28, D-79104 Freiburg, Germany
}

Received March 21; accepted December 13, 1996

\begin{abstract}
In recent years, autoregressive models have had a profound impact on the description of astronomical time series as the observation of a stochastic process. These methods have advantages compared with common Fourier techniques concerning their inherent stationarity and physical background. However, if autoregressive models are used, it has to be taken into account that real data always contain observational noise often obscuring the intrinsic time series of the object. We apply the technique of a Linear State Space Model which explicitly models the noise of astronomical data and allows to estimate the hidden autoregressive process. As an example, we have analysed the X-ray flux variability of the Active Galaxy NGC 5506 observed with EXOSAT.
\end{abstract}

Key words: X-rays: galaxies — methods: statistical galaxies: NGC 5506

\section{Introduction}

A common phenomenon of active galactic nuclei, which presumably harbor supermassive black holes with masses of $10^{6}-10^{9} M_{\odot}$ (Rees 1984), is the strong variability which can be observed in X-ray lightcurves. These AGN lightcurves seem to show featureless "red noise", i.e. scalefree, divergent variability at low frequencies, often also described as flickering or $1 / f$ fluctuation (Lawrence et al. 1987). The $1 / f$ term describes the power law distribution of the spectral power with the function $f^{-\alpha}$ in the power spectrum, often denoted as " $1 / f$ " behavior.

We present an alternative model to analyse the variability seen in the X-ray lightcurves of AGN. The standard method of analyzing time series in the frequency domain is discussed briefly in Sect. 2. The alternative is known as a Linear State Space Model (LSSM) based on the theory of autoregressive processes (Scargle 1981; Honerkamp 1993) which usually cannot be observed directly since the observational noise (i.e. detectors, particle background) overlays the process powering the AGN. A LSSM fit applied to the time series data yields the dynamical parameters of the underlying stochastic process. These parameters should be strongly correlated to the physical properties of the emission process. The corresponding LSSM power spectrum exhibits both the decrease of power at medium frequencies and a limitation of spectral power at low frequencies. The detailed mathematical background of LSSM and the fit procedure are described in Sects. 3 and 4. Finally we present first results using this technique with EXOSAT data from the Seyfert galaxy NGC 5506 in Sect. 5.

\section{Description of the method based on the $1 / f^{\alpha}-$ model}

Although measured astronomical data are time domain data, a commonly applied method works in the frequency domain by analyzing the power spectrum of the time series. As the observational window function is convoluted to the true spectrum of the source, artefacts might be produced in the power spectrum, which make a proper interpretation more difficult (Papadakis \& Lawrence 1995; Priestley 1992). In most cases, the power spectra are fit by a power law function with an offset described as $1 / f^{\alpha}+c$, with values of $\alpha$ ranging from 0 to 2 and a mean of about 1.5 (Lawrence \& Papadakis 1993). The value $c$ is often denoted as the "observational noise floor" which describes the random process comprising the observational errors whereas the "red noise" component is the signal of interest. In the case of long AGN observations, however, a flattening at low frequencies occurs which cannot be modelled by the $1 / f^{\alpha}$-model (McHardy 1988).

The $1 / f^{\alpha}$-model is an ad hoc description of the measured periodogram, without any direct physical motivation. However, it is possible to generate time series with a $1 / f^{\alpha}$-spectrum using self-organized criticality models simulating the mass flow within an accretion disc of the AGN (Mineshige et al. 1994). Such models produce a stationary 
time series that exhibits a $1 / f^{\alpha}$-power spectrum by limiting the timescales occurring in the simulated accretion process. A $1 / f^{\alpha}$-model without limited timescales would be stationary only if the power law slope is smaller than unity (Samorodnitsky \& Taqqu 1994). The observed time series is composed by the superposition of single luminosity bursts. The slope of the $1 / f^{\alpha}$-spectrum of data simulated in that way is about 1.8 , significantly higher than those measured from real data (Lawrence \& Papadakis 1993). If the inclination of the accretion disk is brought in as an additional model parameter the slope can be diminished, but not in a way that leads to convincing results (Abramowicz et al. 1995). Another point that contradicts this assumption is that there is no correlation between the spectral slope and the type of the Seyfert galaxy (Green et al. 1993). This correlation should be present since the Seyfert type is believed to be caused by the inclination of the line of sight (Netzer 1990).

The periodogram which is used to estimate the true source spectrum is difficult to interpret in the presence of non-equispaced sampling time series arising from real astronomical data (Deeter \& Boynton 1982 and references therein). The estimation of the $1 / f^{\alpha}$-spectrum is hampered even in the absence of data gaps. This is due to the finite extent of the observed time series. Therefore, the transfer function (Fourier transform of the sampling function) is a sinc-function which will only recover the true spectrum if this is sufficiently flat (Deeter \& Boynton 1982; Deeter 1984). In the case of "red noise" spectra the sidebands of the transfer function will cause a spectal leakage to higher frequencies which will cause the spectra to appear less steep (the spectral slope will be underestimated).

Even periodograms of white noise time series deviate from a perfectly flat distribution of frequencies as the periodogram is a $\chi_{2}^{2}$-distibuted random variable with a standard deviation equal to the mean (Leahy et al. 1983). Thus the periodograms fluctuate and their variances are independent of the number of data points in the time series. Due to the logarithmic frequency binning, AGN periodograms will always show this strong fluctuation due to the low number of periodogram points averaged in the lowest frequency bins (see Fig. 1).

Furthermore, additional modulations can be created in white noise periodograms if the time series consists of parts which slightly differ in their means and variances, respectively (Krolik 1992). In the case of the EXOSAT ME X-ray lightcurves this effect is due to the swapping of detectors as each detector has its own statistical characteristics which cannot be totally suppressed (Grandi et al. 1992; Tagliaferri et al. 1996). Figure 1a shows a typical X-ray lightcurve which mainly consists of uninterrupted $11 \mathrm{ksec}$ observation blocks before detectors are swapped. If the periodogram frequency corresponds to the observation block length, the calculated sum of Fourier coefficients equals its expected white noise value of $\sigma^{2}$ due to the constant mean and variance within the
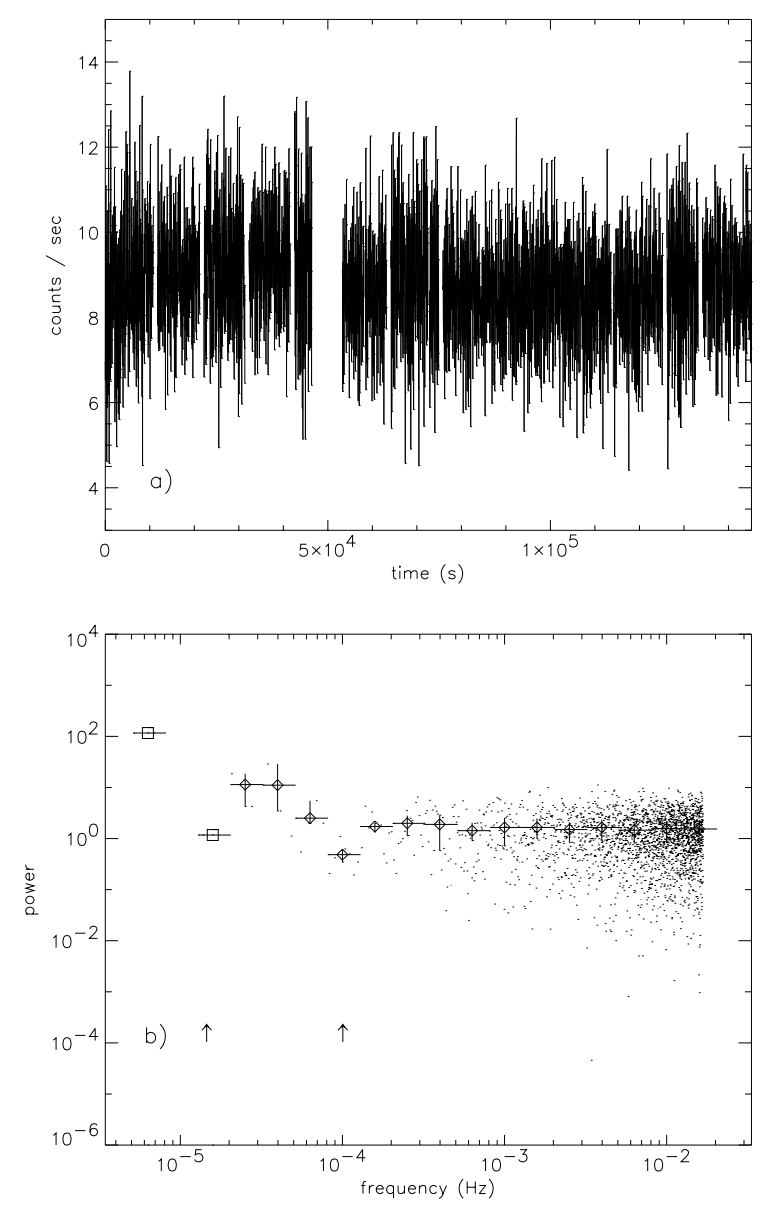

Fig. 1. a) EXOSAT ME X-ray lightcurve of the quasar 3C 273 (Jan. 1986), b) corresponding periodogram. Each dot represents the spectral power at its frequency, stepped with $1 / T_{\text {tot }}$. The periodogram is binned logarithmically (squares indicates a single point within the frequency bin)

entire oscillation cycle. At other, mainly lower, frequencies the Fourier sum yields non-white values due to temporal correlations caused by different means and variances of observation blocks located in the test frequency cycle. These deviations from a flat spectrum will be very strong at frequencies which correspond to twice the observation block length. The arrows in Fig. 1b clearly show this minimum feature at $9.110^{-5} \mathrm{~Hz}$ and another shortage of power at $1.410^{-5} \mathrm{~Hz}$ which corresponds to the long uninterrupted $72 \mathrm{ksec}$ observation block starting at the second half of the EXOSAT observation (Fig. 1a).

Consequently a model is required which operates in the time domain and avoids any misleading systematical effects occuring in power spectra. 


\section{Mathematical background of the linear state} space model

In this section we briefly introduce the Linear State Space Model (LSSM). For a detailed discussion, see Honerkamp (1993) and Hamilton (1995). The LSSM is a generalization of the autoregressive (AR) model invented by Yule (1927) to model the variability of Wolf's sunspot numbers.

We follow Wold's decomposition theorem (Wold 1938; Priestley 1992; Fuller 1996) which states that any discrete stationary process can be expressed as the sum of two processes uncorrelated with one another, one purely deterministic (i.e. a process that can be forecasted exactly such as a strictly period oscillation) and one purely indeterministic. Further, the indeterministic component, which is essentially the stochastic part, can be written as a linear combination of an innovation process, which is a sequence of uncorrelated random variables.

A given discrete time series $x(t)$ is considered as a sequence of correlated random variables. The AR model expresses the temporal correlations of the time series in terms of a linear function of its past values plus a noise term and is closely related to the differential equation describing the dynamics of the system. The fact that $x(t)$ has a regression on its own past terms gives rise to the terminology "autoregressive process" (for detailed discussions see Scargle 1981; Priestley 1992). A time series is thus a realization of the stochastic process or, more precisely, the observation of a realization of the process during a finite time interval. The AR model expresses the temporal correlations in the process in terms of memory, in the sense that a filter $\left(a_{i}\right)$ remembers, for a while at least, the previous values $x(t-i)$. Thus the influence of a predecessor value decreases as time increases. This fading memory is expressed in the exponential decay of the AR autocorrelation function (see Eq. 10). The AR processes variable $x(t)$ remembers its own behavior at previous times, expressed in a linear relationship in terms of $x(t-1), x(t-2), \ldots$ plus $\epsilon(t)$ which stands for an uncorrelated (Gaussian) white noise process.

$x(t)=\sum_{i=1}^{p} a_{i} x(t-i)+\epsilon(t), \quad \epsilon(t) \sim N\left(0, \sigma^{2}\right)$.

The number of terms $p$ used for the regression of $x(t)$ determine the order of the AR process, which is abbreviated to an $\operatorname{AR}[\mathrm{p}]$ process. The parameter values $a_{i}$ have to be restricted for the process to be stationary (Honerkamp 1993). For a first order process this means $\left|a_{1}\right|<1$, for a second order process: $\left|a_{1} \pm \sqrt{a_{1}^{2}+4 a_{2}^{2}}\right|<2$. Depending on the order $p$, the parameters $a_{i}$ of the process represents damped oscillators, pure relaxators or their superpositions. For the first order process AR[1] the relaxation time $\tau$ of the system is determined from $a_{1}$ by:

$\tau=-\frac{1}{\log \left|a_{1}\right|}$.
In the case of a damped oscillator for an $\mathrm{AR}[2]$ process the parameters, the period $T$ and the relaxation time $\tau$ respectively, are related by:

$a_{1}=2 \cos \left(\frac{2 \pi}{T}\right) \mathrm{e}^{-1 / \tau}$

$a_{2}=-\mathrm{e}^{-2 / \tau}$.

For a given time series the parameters $a_{i}$ can be estimated e.g. by the Durbin-Levinson- or Burg-algorithm (Honerkamp 1993). By statistical testing it is possible to infer whether a model is compatible with the data.

A first generalization of AR models are the autoregressive-moving-average (ARMA) models that include also past noise terms in the dynamics:

$x(t)=\sum_{i=1}^{p} a_{i} x(t-i)+\sum_{j=1}^{q} b_{j} \epsilon(t-j)+\epsilon(t)$.

Both models, AR and ARMA processes, assume that the time series is observed without any oberservational noise. In presence of such noise the parameters $a_{i}$ will be underestimated and statistical tests will reject the model even if its order is specified correctly.

LSSMs generalize the AR and ARMA processes by explicitly modelling observational noise. Furthermore, LSSMs use the so called Markov property, which means that the entire information relevant to the future or for the prediction is contained in the present state. The variable $x(t)$ that has to be estimated cannot be observed directly since it is covered by observational noise $\eta(t)$. Following the Markov property it is possible to regressively predict the values $x(t)$, though.

The measured observation variables $y(t)$ may not necessarily agree with the system variables $x(t)$ that provide the best description of the system dynamics. Thus a LSSM is defined with two equations, the system or dynamical Eq. (6) and the observation Eq. (7).

$$
\begin{aligned}
\boldsymbol{x}(t) & =\mathrm{A} \boldsymbol{x}(t-1)+\boldsymbol{\epsilon}(t) \quad \boldsymbol{\epsilon}(t) \sim N(0, \mathrm{Q}) \\
y(t) & =\mathrm{C} \boldsymbol{x}(t)+\eta(t) \quad \eta(t) \sim N(0, R) .
\end{aligned}
$$

This definition is a multivariate description, which means that the $\mathrm{AR}[\mathrm{p}]$ process is given as a $p$-dimensional AR process of order one, with a matrix $A$ that determines the dynamics. By combining the different dimensional terms of the multivariate description the typical AR $[\mathrm{p}]$ (see Eq. 1) form can be derived easily. The observation $y(t)$ is formulated as a linear combination of the random vectors $\boldsymbol{x}(t)$ and $\eta(t)$. The matrix $C$ maps the unobservable dynamics to the observation. The terms $\boldsymbol{\epsilon}(t)$ and $\eta(t)$ represent the dynamical noise with covariance matrix $Q$ and the observational noise with variance $R$, respectively.

The estimation of the parameters in LSSMs is more complicated than for AR or ARMA processes. There are two conceptually different procedures available to obtain 
predictions of the Kalman filter plus a white noise realization with the original lightcurves variance. Generally, gaps in an observed time series can be handled by the LSSM in a natural way avoiding the filling of gaps with Poisson noise. The key is again the Kalman filter. The Kalman filter considers the fact that there are still decaying processes taking place even if the object is not observed. In each cycle of the iterative parameter estimation procedure $\boldsymbol{x}(t)$ is estimated based on an internal prediction, corrected by information obtained from the actual data $y(t)$. In case of gaps no information from $y(t)$ is available and the internal prediction decays in its intrinsic manner until new information is given. In the case of the lightcurve of NGC 5506 the resulting parameters are consistent with those of the first approach due to the high duty cycle of the original time series.

\section{The EXOSAT observation of NGC 5506}

As the X-ray lightcurves from EXOSAT are the longest AGN observations available, we have used the longest individual observation of about $230 \mathrm{ks}$ of the Seyfert galaxy NGC 5506 for applying the LSSM (Fig. 2a). The data which have been extracted from the HEASARC EXOSAT ME archive, are background subtracted and dead time corrected, with a $30 \mathrm{~s}$ time resolution obtained over $1-8 \mathrm{keV}$ energy range. The Seyfert galaxy NGC 5506 holds a special place in AGN variability studies, as it is both bright and one of the most variable AGN. The chosen lightcurve contains only few gaps providing a duty cyle of $92.4 \%$. The mean and rms of the lightcurve are 6.87 and 1.55 counts in $30 \mathrm{~s}$ bins.

Table 1. Results of LSSM fits to the EXOSAT NGC 5506 data

\begin{tabular}{ccccc}
\hline $\begin{array}{c}\text { Model } \\
\text { LSSM AR[p] }\end{array}$ & $R_{\eta}^{a}$ & $\begin{array}{c}\text { Periods } \\
(\mathrm{s})\end{array}$ & $\begin{array}{c}\tau^{b} \\
(\mathrm{~s})\end{array}$ & $\begin{array}{c}\text { KS test }^{c} \\
.\end{array}$ \\
\hline 0 & 1 & - & - & $0.0 \%$ \\
1 & 0.722 & - & 4799 & $93.5 \%$ \\
2 & 0.701 & - & 26.1 & $66.8 \%$ \\
& & - & 5011 & \\
3 & 0.510 & - & 10.6 & $88.2 \%$ \\
& & - & 18.9 & \\
4 & & - & 4798 & \\
& 0.395 & 236.3 & 71.1 & $92.1 \%$ \\
& & - & 6.7 & \\
& & - & 4780 &
\end{tabular}

\footnotetext{
${ }^{a}$ Variance of the observational noise.

${ }^{b}$ Relaxation time.

${ }^{c}$ Kolmogorov-Smirnov test for white noise.
}

We applied LSSMs with different order AR processes. An LSSM using an AR [0] process corresponds to a pure white noise process without any temporal correlation and a flat spectrum. The used Kolmogorov-Smirnov test rejects this model at any level of significance (see Table 1). Without loss of generality, $Q$ is set to unity, the mean and variance are set to 0 and 1 , respectively. We see that the X-ray lightcurve of NGC 5506 can be well modelled with a LSSM AR[1] model, as the residuals between the estimated AR[1] process and the measured data are consistent with Gaussian white noise. Figure 3 shows the distribution and the corresponding normal quantile plot of the fit residuals which both display the Gaussian character of the observational noise. The standard deviation of the distribution is 0.738 which is in good agreement to the estimated observational variance of 0.722 for the LSSM AR[1] fit (see Table 1). Furthermore, the lightcurve of the estimated AR[1] looks very similar to the temporal behavior of the hidden process (Fig. 2). The corresponding dynamical parameter $a_{1}$ of the LSSM AR[1] fit is 0.9938 which corresponds to a relaxation time of about $4799 \mathrm{~s}$.
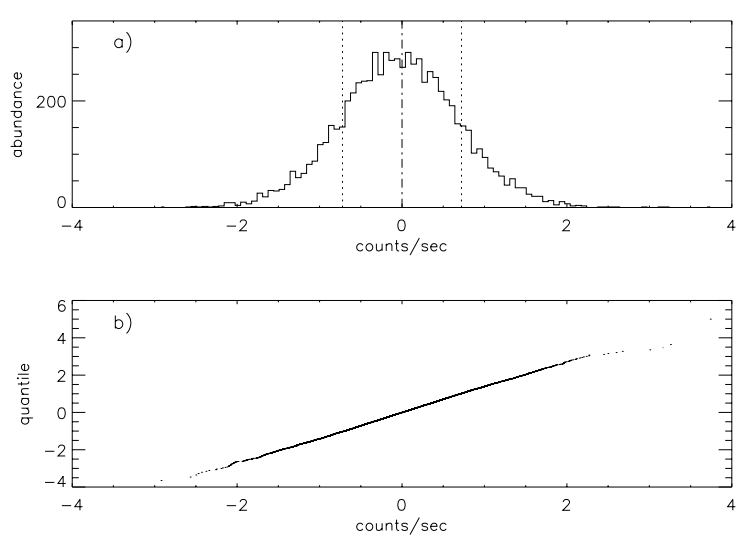

Fig. 3. a) Distribution and b) normal quantile plot of the residuals of the LSSM AR[1] fit to the EXOSAT ME NGC 5506 lightcurve (the dotted lines in a) indicate the mean and rms of the observational noise). A normal quantile plot arranges the data in increasing order and plot each data value at a position that corresponds to its ideal position in a normal distribution. If the data are normally distributed, all points should lie on a straight line

The LSSM AR[1] gives a good fit to the EXOSAT NGC 5506 data as the variance of the prediction errors nearly remains constant from model order 1 to 2 and the residuals conforms to white noise. The decrease in the variance for higher model orders might be due to correlations in the modelled noise, generated by the switching of the EXOSAT detectors. Since each detector has its own noise charateristics a regular swapping between background and source detectors would lead to an alternating observational noise level (see Sect. 2). The higher order LSSM $\mathrm{AR}[\mathrm{p}]$ fits try to model the resulting correlations with additional but negligible relaxators and damped oscillators $\left(\tau \approx\right.$ bintime, $\left.\tau \ll T_{\text {tot }}\right)$. 
We have used the Durbin-Levinson algorithm (see Sect. 3) to estimate the parameters of a competing simple AR[p] model (see Table 2). As expected for time series containing observational noise, the characteristic timescales are underestimated by fitting a simple AR process and the statistical test rejects the AR[p] model. A test for white noise residuals fails, which means that there are still correlations present which cannot be modelled with an $\mathrm{AR}[\mathrm{p}]$ procces. We have performed $\mathrm{AR}[\mathrm{p}]$ fits for model orders up to 10 and we never found residuals consitent with white noise, indicating that there is no preferred model order. All occuring relaxators and damped oscillators are insignificant due to their short relaxation timescales compared with the bintime of $30 \mathrm{~s}$. As the observational noise is not modelled explicitly in AR models, it is included accidentally in the inherent AR noise term. Thus, any correlation in the observed time series which can be detected in the LSSM fits, is wiped out and the higher order AR fits only reveal fast decaying relaxators and oscillators.

Table 2. Results of AR fits to the EXOSAT NGC 5506 data

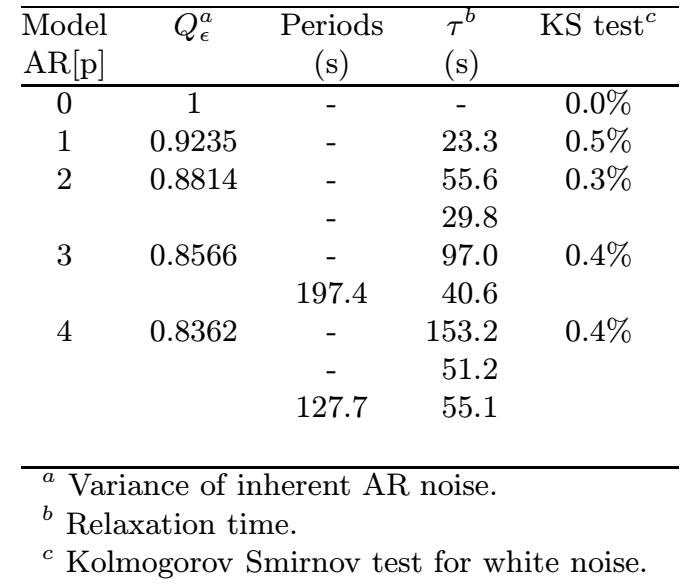

One might expect that the resulting best fit LSSM light curve (Fig. 2b) might also be produced by just smoothing the original lightcurve. This assumption is wrong as a smoothing filter would pass long timescales and suppress all short time variability patterns. Thus all information about the variations on short timescales would be lost (Brockwell \& Davis 1989). The Kalman filter concedes not only the time series values $x(t)$ but also its prediction errors. These errors are much smaller than the errors of the observed lightcurve $y(t)$. In the case of the NGC 5506 observation (Fig. 2) the estimation errors are about 0.18 counts/s and the errors of $y(t)$ are about 1.3 counts $/ \mathrm{s}$, respectively. Both lightcurves in Fig. 2 are shown without error bars due to reasons of clarity.

We have used Monte Carlo Simulations to determine the error of the dynamical parameter $a_{1}$. Using the dis- tribution of the estimated parameters of 1000 simulated $\mathrm{AR}[1]$ time series with the best fit results, we found $a_{1}=0.9938 \pm 0.0007$. As the dynamical parameter is close to unity the corresponding relaxation time error is high, with $\tau=4799_{-472}^{+632}$ s. To prove the quality of the LSSM results we have fitted a LSSM AR[1] spectrum to the periodogram data. This fit yields the dynamical parameter $a_{1}=0.9936 \pm 0.0021$ which is consistent with the LSSM AR[1] fit in the time domain, but the corresponding error is much higher due to the lower statistical significance of frequency domain fits (see Sect. 2).

The autocovariance function of the $\mathrm{AR}[1]$ process is given by:

$\operatorname{ACF}_{\mathrm{AR}[1]}(\Delta)=\frac{Q}{1-a_{1}^{2}} \mathrm{e}^{\log \left(a_{1}\right) \Delta}$

which is an exponentially decaying function for stationary $\left(\left|a_{1}\right|<1\right)$ time series, very similar to the temporal behavior of the autocorrelation function of a shot noise model (Papoulis 1991):

$\operatorname{ACF}_{\text {shot noise }}(\Delta)=\frac{\lambda \tau}{2} \mathrm{e}^{-\Delta / \tau}$.

The variable $\lambda$ denotes the density and $\tau$ is the lifetime of the shots. This similarity means that an $\mathrm{AR}[1]$ process can also be modelled by a superposition of Poisson distributed decaying shots (Papoulis 1991). The shot noise model, which has been used as an alternative to the $1 / f^{\alpha}$ model, appears to give a good fit to the power spectrum of NGC 5506 (Papadakis \& Lawrence 1995; Belloni \& Hasinger 1990 and references therein). But instead of all the shots having the same lifetime, Papadakis \& Lawrence (1995) used a distribution varying as $\tau^{-2}$ between $\tau_{1}$ and $\tau_{2}$. They fixed $\tau_{2}$ arbitrarily at $12000 \mathrm{~s}$ and found that $\tau_{1}$ is around $300 \mathrm{~s}$ for NGC 5506, much lower than the relaxation time of about $4800 \mathrm{~s}$ found with the LSSM fit. A possible explanation for this difference could be the distribution of lifetimes. Since the power law slope of the shot noise model is constantly -2 at medium and high frequencies, this distribution is necessary to modify the slope and to maintain a good fit to the spectrum. The advantage of a LSSM is a variable slope at medium frequencies which depends on the dynamical parameter (see Fig. 4).

The shot noise model can be regarded as an approximation of an AR[1] model for values $a_{1}$ near unity. The mean density of the Poisson events $\lambda$ then corresponds to the variance $Q$ of the dynamical noise in the LSSM system Eq. (6). Thus $Q$ could be used to quantify and compare the rate of the accretion shots occuring in AGNs.

\section{Discussion}

We obtain a convincing fit to the observed X-ray lightcurve of an AGN using a LSSM AR[1] process as well in the time and in the frequency domain. The explicit modelling of observational noise allows to estimate 


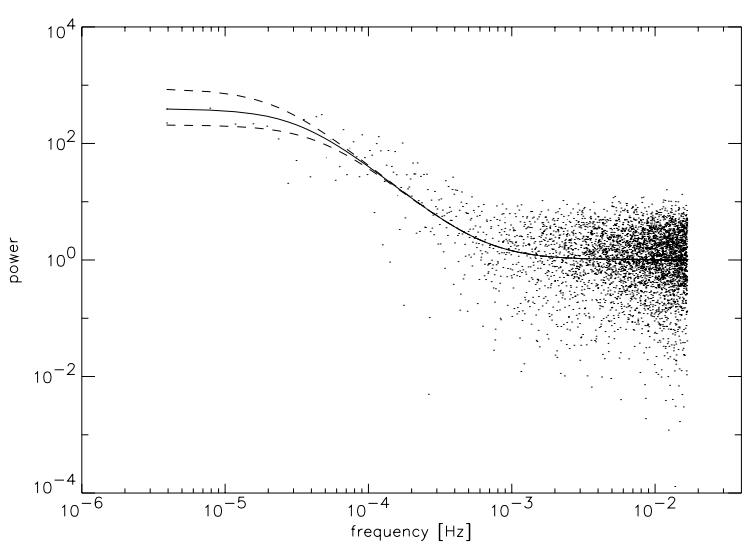

Fig. 4. Periodogram of the EXOSAT ME X-ray lightcurve of NGC 5506 (dots) and the spectrum of the best fit LSSM AR[1] model in the time domain (line) (see Fig. 2a). The spectra of the higher order LSSM AR fits differ less than $2 \%$ from the LSSM AR[1] spectrum. The dashed lines display the $\pm 1 \sigma$ - spectra of the corresponding frequency domain fit. The time domain fit yields $1 \sigma$ errors which are more than 3 times smaller (see text for details)

the covered $\mathrm{AR}[1]$ process, indicating that the stochastic process is dominated by a single relaxation timescale. We show that the general AR[p] model (see Eq. 1) can be restricted to a simple $\mathrm{AR}[1]$ process which succeeds in describing the entire dynamics of the observed AGN X-ray lightcurve.

It has been suggested by McHardy (1988) that the single shots, which are supposed to be superimposed to build the lightcurve, may arise from subregions of an overall larger chaotic region which are temporarily lit up, perhaps by shocks. Since one would expect a non uniform electron density throughout this region (probably decreasing with distance from the central engine), the resulting difference in cooling timescales yields the different decay timescales (Green et al. 1993). As the LSSM predicts that the stochastic process is dominated by a single relaxator, we presume the existence of a single cooling timescale or a uniform electron density in the emission region following the shot noise model (see Sutherland et al. 1978).

The assumption of an exponentially decaying shot seems to be reasonable as time-dependent Comptonisation models lead to such a pulse profile. The scenario for a thermal Comptonisation model (Payne 1980; Liang \& Nolan 1983) starts with UV photons which arise as the accretion inflows inhomogeneities, each producing a single flare when gravitational energy is set free as radiation. The impulsive emission of the Poisson distributed delta peaks in a cloud of hot electrons triggers X-ray flares with a specific pulse profile depending on the seed photon energy, the density, and the temperature of the electrons. This impulsive emission is delayed and broadened in time and spectrally hardened due to repeated Compton scattering.
Some approximate analytic solutions of this process show that the temporal evolution of the generated X-ray pulse can be described by a nearly exponentially decaying function (Miyamoto \& Kitamoto 1989). The only difference to the "shots" used above is the (more realistic) non-zero rise time. Using this model it should be possible to associate the estimated relaxator timescale $\tau_{\mathrm{AR}[1]}$ with the physical properties of the Comptonisation process.

The presented LSSM can also be used to analyse X-ray variability of galactic X-ray sources. As both, relaxators and (damped) oscillators can be estimated, it is possible to use the algorithm to search for periodocities and QPO phenomena in the lightcurves of X-ray binaries (see Robinson \& Nather 1979; Lewin et al. 1988; van der Klis 1989).

Acknowledgements. We would like to thank J.D. Scargle, R. Staubert, M. Maisack, J. Wilms and K. Pottschmidt for helpful discussions and C. Gantert for writing the code of the LSSM program. Furthermore, we thank the anonymous referee for constructive comments.

\section{References}

Abramowicz A.R., Chen X., Kato S., et al., 1995, ApJL 438, L37

Begelman M.C., de Kool M., 1991, in Variability in Active Galactic Nuclei, Miller H.R. and Wiita P.J. (eds). Cambridge: Cambridge Univ. Press, p. 198

Belloni T., Hasinger G., 1990, A\&A 227, L33

Brockwell P.J., Davis R.A., 1991, Time Series: Theory and Methods. Springer Verlag, 2nd. ed.

Deeter J.E., 1984, ApJ 281, 482-491

Deeter J.E., Boyton P.E., 1982, ApJ 261, 337-350

Fuller W.A., 1996, Introduction to Statistical Time Series, New York. John Wiley, 2nd. ed.

Grandi P., Tagliaferri G., Giommi P., et al., 1992, ApJS 82, 93

Green A.R., McHardy I.M., Lehto H.J., 1993, MNRAS 265, 664-680

Hamilton J.D., 1995, Time Series Analysis. Princeton University Press

Honerkamp J., 1993, Stochastic Dynamical Systems. VCH Publ. New York, Weinheim

Koen C., Lombard F., 1993, MNRAS 263, 287

Krolik J.H., 1992, Statistical Challenges in Modern Astronomy, Feigelson E. and Babu G.J. (eds.). Springer Verlag New York, p. 349

Lawrence A., Watson M.G., Pounds K.A., et al., 1987, Nat 325,694

Lawrence A., Papadakis P., 1993, ApJS 414, 85

Leahy D.A., Darbro W., Elsner R.F., Weisskopf M.C, Sutherland P.G., 1983, ApJ 266, 160-170

Lehto H.J., 1989, in Proc. 23rd ESLAB Symp. on Two Topics in X-ray Astronomy, Vol. 1, Hunt J. and Battrick B. (eds.). ESA SP-296, Noordwijk, p. 499

Lewin W.H.G., van Paradijs J., van der Klis M., 1988, Space Sci. Rev. 46, 273

Liang E.P., Nolan P.L., 1983, Space Sci. Rev. 38, 353

McHardy I., Czerny B., 1987, Nat 325, 696 
Mineshige S., Ouchi N.B., Nishimori H., et al., 1994, PASJ 46, Robinson E.L., Nather R.E., 1979, ApJS 39, 461 97

Miyamoto S., Kitamoto S., 1989, Nat 342, 773

Netzer H., 1990, Saas-Fee Advanced Course 20 on Active Galactic Nuclei, Blandford R.D., Netzer H., Woltjer L. (eds.) p. 57-160

Papadakis I.E., Lawrence A., 1995, MNRAS 272, 161

Papoulis A.P., 1991, Probability, Random Variables and Stochastic Processes. New York, McGraw-Hill, 3rd. ed.

Payne D.G., 1980, ApJ 232, 951

Priestley M.B., 1992, Spectral Analysis and Time Series. San Diego, Academic Press

Rees M.J., 1984, ARA\&A 22, 471

Samorodnitsky G., Taqqu M.S., 1994, Stable Non-Gaussian Random Processes. New York, Chapman and Hall

Scargle J.D., 1981, ApJS 45, 1

Sutherland P.G., Weisskopf M.C., Kahn S.M., 1978, ApJ 219, 1029

Tagliaferri G., Bao G., Israel G.L., et al., 1996, ApJ (accepted for publication)

van der Klis M., 1989, ARA\&A 27, 517

Wold H.O.A., 1938, A Study in the Analysis of Stationary Time Series. Uppsala, Almqvist and Wiksell, 2nd. ed.

Yule G., 1927, Phil. Trans. R. Soc. A 226, 267 